\title{
Agile Methoden als Moderatoren zur Steuerung der sozialen Beschleunigung
}

\author{
Bertil Haack*, Markus Müller-Trabucchi
}

\section{Zusammenfassung}

Das Gefühl, unter Zeitdruck zu stehen und unter Zeitnot zu leiden, ist zumindest in der westlichen Welt weit verbreitet. Klassisches Zeitmanagement kann als Mitverursacher für die Zeitverdichtung gesehen werden und sollte daher sinnvoll modifiziert bzw. ausgebaut werden. Hierzu bietet sich die Theorie der sozialen Beschleunigung als Ausgangspunkt an (Rosa 2012). Sie liefert eine Erklärung für die Zeitverdichtung und bringt die Autoren zu dem Vorschlag, die soziale Beschleunigung mittels sogenannter Moderatoren zu steuern. Ergebnisse aus Zeitmanagement-Workshops legen die Vermutung nahe, dass agile Arbeitsmethoden als Moderatoren zur Steuerung der sozialen Beschleunigung geeignet sein könnten, und führen die Autoren zu einem Konstruktionsschema für derartige Moderatoren.

\begin{abstract}
In the Western world, the feeling of being under time pressure and suffering from a shortage of time is widely experienced. Classical time management can be seen as a contributing factor for the time compaction and should, therefore, be modified or extended, with the theory of social acceleration as the starting point (Rosa 2012). This theory provides an explanation for the time compression and leads us to the proposal that so-called moderators can control the social acceleration. Results from time management workshops suggest that agile working methods can be used as moderators to control social acceleration, and lead us to a design scheme for such moderators.
\end{abstract}

\section{Einleitung: Von verlorener und verdichteter Zeit}

Wem kommt nicht aus eigenem Erleben bekannt vor, was Florian Opitz in der Einleitung seines Buches „Speed - Auf der Suche nach der verlorenen Zeit“ (Opitz 2011) so eindrücklich beschreibt: „Ich hatte eigentlich immer das Gefühl, ein ganz normales und erfülltes Leben zu führen. [...] Doch irgendwie habe ich in den letzten Jahren gemerkt, dass etwas mit mir nicht stimmt. Ich habe keine Zeit. [...] Es ist jedes Mal das Gleiche: Ein Job ist erledigt, ein neues Jahr beginnt, und ich nehme mir vor, dass ab jetzt alles anders wird. Endlich mal wieder ausgehen, Zeit mit Freunden oder der Familie verbringen, ins Kino oder zu Konzerten gehen. Doch dann dauert es nicht lange, und meine Pläne zerplatzen wie Seifenblasen. Und ich hetze wieder genauso atemlos durchs Leben wie zuvor. [...] Meine Erfahrung mit der Zeit beschränkt sich inzwischen nur auf das eine Gefühl - sie fehlt“ (Opitz 2011: 7ff.).

Wo aber bleibt die Zeit? Eine - zumal verblüffend einfache - Antwort lässt sich leicht geben: Man nutzt die durch die technischen Möglichkeiten - beispielsweise in Form von E-Mail-Systemen, immer schnelleren Rechnern und immer schnelleren Internetverbindungen - bestehenden Möglichkeiten gerade nicht dazu, ,nur' die gleiche Anzahl von Korrespondenzvorgängen wie ohne sie zu erledigen und die damit frei werdende Zeit für unerfüllten Wünsche einzusetzen. Stattdessen dient sie einem - um im Bild zu bleiben - dazu, mehr Korrespondenzvorgänge als vorher zu erledigen (vgl. Haack 2015: 241, Haack \& Müller-Trabucchi 2016: 19). Man „verdichtet“ (Rosa 2015: 42) die eigene Zeit, „die freigesetzten Zeitressourcen werden durch paralleles und häufig sogar exponentielles Mengen- wachstum wieder aufgefressen" (Rosa 2015: 42). Zeitdruck und Zeitnot nehmen spürbar sowohl im beruflichen als auch im privaten Kontext zu.

Der vorliegende Artikel gibt Antworten auf die Frage, wie mit dieser Zeitverdichtung umgegangen werden kann und wie ein Ausweg aus der Zeitnot aussehen könnte. Konkret wird argumentiert, dass das klassische Zeitmanagement als Mitverursacher für die Zeitverdichtung gesehen werden kann und Zeitmanagementmethoden daher sinnvoll modifiziert bzw. ausgebaut werden sollten: Beyond Zeitmanagement (Kapitel 2). Hierzu bietet sich die von Hartmut Rosa entwickelte Theorie der sozialen Beschleunigung als Ausgangspunkt an (vgl. Rosa 2012). Sie liefert eine Erklärung für die Zeitverdichtung in allen - beruflichen wie privaten - Lebensbereichen (Kapitel 3) und bringt uns zu dem Vorschlag, die soziale Beschleunigung in diesen Feldern mittels sogenannter Moderato- 
ren zu steuern (Kapitel 4). Ergebnisse aus Zeitmanagement-Workshops mit Technischen Redakteuren legen den Schluss nahe, dass sich agile Arbeitsmethoden als Moderatoren zur Steuerung der sozialen Beschleunigung eignen können, und führen zu einem Konstruktionsschema für derartige Moderatoren (Kapitel 5). Fazit und Ausblick beschließen die Ausführungen (Kapitel 6).

\section{Beyond-Zeitmanagement}

Klassische Zeitmanagementansätze wie man sie etwa bei Seiwert (2005) findet, sind selbst nach ihrer ,Modernisierung' (vgl. Seiwert 2009, 2013) nicht unbedingt hilfreich, gegen die Zeitverdichtung anzugehen, sondern tragen vielmehr noch zur Zeitarmut bei: Ihnen geht es nicht darum, die mit ihrer Hilfe ,eingesparte' Zeit tatsächlich zur Minderung des Zeitdrucks zu nutzen, sondern darum, wie diese ,freie' Zeit zur Erhöhung unserer Arbeitsleistung eingesetzt werden kann. Wenn man der Zeitnot ernsthaft begegnen will, muss man demnach ein anderes Konzept als klassisches Zeitmanagement für den Umgang mit der eigenen Zeit finden. Konkret geht es den Autoren somit darum, Ideen für ein modernes Zeitmanagement zu entwickeln, das die nützlichen Komponenten des klassischen Zeitmanagements, wie beispielsweise das Eisenhower-Prinzip (vgl. Covey 2014: 182), integriert, das aber darüber hinausreicht, indem es dessen "sehr eindimensionale Ausrichtung auf das ,Immer-schneller-sein-Wollen` überwindet, ohne die ökonomischen Belange und Zielsetzungen der anwendenden Organisation aus dem Auge zu verlieren" (Haack et al. 2016: 21): Beyond Zeitmanagement! Hierzu bietet sich die von Hartmut Rosa entwickelte Theorie der sozialen Beschleunigung als Ausgangspunkt an (vgl. Rosa 2012, 2014, Kodalle \& Rosa 2008, King \& Gerisch 2009). Sie liefert eine Erklärung für die Zeitverdichtung in jedwedem menschlichen Handlungsfeld und führt entsprechend zum Vorschlag, die soziale Beschleunigung mittels sogenannter Moderatoren zu steuern.

\section{Zeitverdichtung durch soziale Beschleunigung}

Nach Hartmut Rosa (2012: 243ff.) leben wir in einer durch die beschleunigte Umwandlung von Verhältnissen, Institutionen und Beziehungen geprägten Welt - sprich: in einer Welt sozialer Beschleunigung. Die soziale Beschleunigung stellt sich dabei als eine Mischung von

- technischer Beschleunigung,

- Beschleunigung des sozialen Wandels sowie

- Beschleunigung des Lebens-/ Arbeitstempos

dar, die einerseits jeweils einen eigenen Motor als Antrieb besitzen und die sich andererseits ohne Unterlass wie in Abb. 1 dargestellt beschleunigen. Dies kann als Ursache der stets voranschreitenden Zeitverdichtung und damit von Zeitdruck und -not gesehen werden.

Zur Erklärung legen die Autoren den Fokus auf die Wirtschaft als Teilelement der Gesellschaft und beginnen mit der technischen Beschleunigung: Historische Untersuchungen zeigen, dass der Zusammenhang ,Zeit ist Geld' schon sehr frühzeitig gesehen und deshalb zum steten Motor für technische Entwicklung und Beschleunigung wurde. Beispielsweise war Vertretern des in Augsburg ansässigen schwäbischen Kaufmannsgeschlechts der Fugger bereits im 16. Jahrhundert klar, dass ein Zeitvorsprung einen Wettbe- werbsvorsprung bedeutet und damit die Möglichkeit bietet, wirtschaftlich erfolgreicher als die Konkurrenten zu sein. Entsprechend ersetzten sie die in ihrer Zeit üblichen Ratsboten durch ein Stafettensystem, mit dem sie ihre kaufmännischen Daten schneller als ihre Wettbewerber übertragen konnten (Haack 2015: 252f.).

Weitere Exempel insbesondere aus der Gegenwart verdeutlichen, dass die technische Beschleunigung aber nicht nur in Geld zählbare Effekte bedingt. Sie ist zudem eine wesentliche Ursache für einen beschleunigten Wandel des sozialen Miteinanders von Menschen sowohl in ihren beruflichen als auch in ihren privaten Umfeldern. So führen Computer, Smartphones und Internet in immer schnellerer Abfolge „u. a. zu neuen Arbeitsstrukturen (Beispiel: virtuelle Projektteams), neuen Produktionsweisen (Beispiel: 3D-Druck), veränderten Kommunikationsstrukturen (Beispiel: E-Mail), veränderten Beziehungsstrukturen (Beispiel: soziale Netzwerke) und neuen Formen sozialer Identität (Beispiel: Avatare in virtuellen Lebensräumen wie etwa Second Life)“ (Haack 2015: 257f.). Mit Hilfe der Technik wird die Gesellschaft, in der wir leben, mehr und mehr zu einer 24/7-Non-Stopp-Gesellschaft. Nahezu jeder Service kann zu jeder Zeit via Internet in Anspruch genommen werden. Wartezeiten, etwa bis ein Büro oder ein Geschäft am nächsten Morgen wieder öffnet, werden so zum einen in Richtung null reduziert. Zum anderen geht dadurch umgekehrt aber auch die Bereitschaft vieler Menschen, auf irgendetwas oder

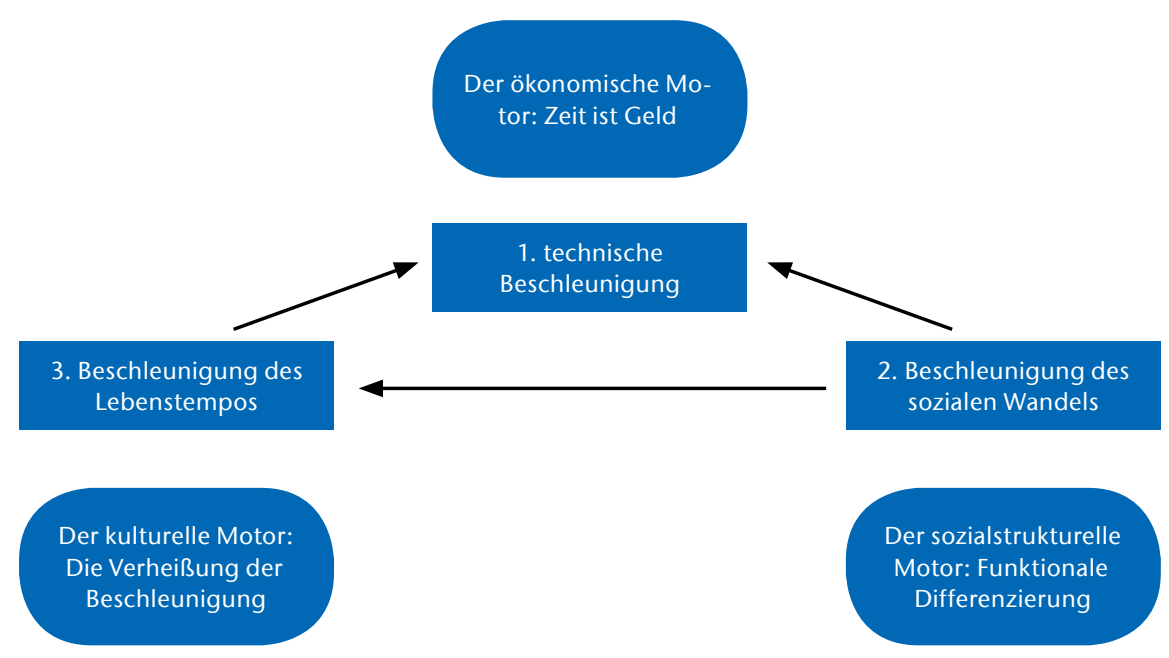

Abb. 1) Der Akzelerationszirkel der sozialen Beschleunigung und seine externen Triebkräfte (Eigene Darstellung in Anlehnung an Rosa 2012: 309). 
irgendwen warten zu wollen, gegen null (Haack 2015: 253f.).

Für den in dieser von beschleunigtem technischem und sozialem Wandel geprägten Gesellschaft agierenden Mensch bedeuten die bisherigen Ergebnisse, dass er mit den Anforderungen dieser Gesellschaft nur mithalten kann, wenn er schnell genug ist, d. h., wenn er sein Lebenstempo durch schnelleres Handeln erhöht. Hier ist der Ruf nach besserer - gemeint ist insbesondere: schnellerer - Technik offenkundig (vgl. Rosa 2012: 244, Haack 2015: 258). Damit ist man aber wieder am Ausgangspunkt der ,technischen Beschleunigung' angekommen. Man erkennt, dass die o. g. drei Dimensionen der sozialen Beschleunigung einen geschlossenen, sich selbst antreibenden und immer schneller werdenden "Akzelerationszirkel“ (Rosa 2012: 251) bilden: Die technische Beschleunigung führt zur Beschleunigung des sozialen Wandels, diese fordert wiederum die Beschleunigung des Lebenstempos, die sich grundsätzlich nur durch technische Beschleunigung realisieren lässt (s. Abb. 1). „Einmal in Bewegung gesetzt, kommt dieser Akzelerationszirkel nicht mehr zur Ruhe, sondern erzeugt stets und unvermeidlich mehr Beschleunigung bis hin zum rasenden Stillstand“ (Haack 2015: 267).

Ebenso wie die technische Beschleunigung besitzen auch die anderen beiden Dimensionen sozialer Beschleunigung eigene Antriebe, die zusätzliche Beschleunigungsimpulse für die jeweilige Dimension und damit für den Akzelerationszirkel liefern. Im Falle der Beschleunigung des sozialen Wandels handelt es sich um das Prinzip der funktionalen Differenzierung, d. h. um „Prozesse der zunehmenden Arbeitsteilung bzw. der wachsenden Ausdifferenzierung von Funktionsund Wertsphären: Die Wirtschaft, die Wissenschaft, die Kunst: sie alle folgen als solche ausdifferenzierten Sphären ihren eigenen Gesetzen und Logiken, ohne noch durch eine klare politische oder religiöse Steuerungsinstanz zusammengehalten zu werden" (Rosa et al. 2013: 23). Bestand der Nutzen dieses Prinzips zunächst darin, dass der handelnde Mensch seine einzelnen Aufgaben in den jeweiligen Aufgabenbereichen weitgehend ungestört und damit zügiger als ohne deren Ausdifferenzierung bearbeiten konnte, führt es nunmehr umgekehrt zu einer fortwährenden Verstetigung aller Prozesse in Richtung Non-Stopp-Gesellschaft (s. o.) und trägt damit zur Beschleunigung des sozialen Wandels bei. Ein ähnliches Bild zeigt sich auch im Falle der Beschleunigung des Lebenstempos. Möchte man annehmen, dass die Beschleunigung des Lebenstempos grundsätzlich eher negativ gesehen wird, wirkt beispielsweise das Arbeiten unter Zeitdruck demgegenüber durchaus für viele Menschen wie eine Art Droge mit entsprechenden Abhängigkeitseffekten. Zufriedenheit (sofern dieser Begriff hier überhaupt gerechtfertigt ist) stellt sich bei ihnen nur ein, wenn sie dem Zeitdruck standhalten und alle Aufgaben erledigen können - ein Ziel, das innen durch immer schnelleres Handeln, also durch eine fortwährende Beschleunigung des Lebenstempos, realisierbar erscheint und das daher wie eine Verheißung der Beschleunigung wirkt (Haack 2015: 253ff.).

\section{Die soziale Beschleunigung moderieren}

Festzuhalten sind einige Aspekte, die aus dem Modell des Akzelerationszirkels der sozialen Beschleunigung resultieren:

- Die Zeitverdichtung nimmt offenbar zu bzw. ab, je nachdem, ob der Akzelerationszirkel mehr oder weniger Beschleunigung erzeugt.

- Es kann nicht darum gehen, dass der Akzelerationszirkel zum Stillstand kommt. Dies bedeutete nämlich beispielsweise, dass man sich der technischen Modernisierung verschlösse - ein sicher nicht gewünschter Zustand.

- Es kann aber auch nicht darum gehen, den Akzelerationszirkel ungebremst laufen zu lassen. Dies bedeutete nämlich, ein Maß an sozialer Beschleunigung und damit ein Maß an Zeitverdichtung zuzulassen, dem Menschen auf Dauer garantiert nicht mehr gewachsen wären (vgl. Haack 2015: 267ff.).
Modernem Zeitmanagement muss es also in jeder gegebenen Situation darum gehen, den Akzelerationszirkel so zwischen den Status ,Stillstand" und ,ungebremst' zu steuern, dass er ähnlich wie ein Stromgenerator immer die angemessene, erforderliche Strommenge - nicht mehr und nicht weniger - und damit auch eine akzeptable Begründung für die jeweils zugehörige Zeitverdichtung bereitstellt. Dies bedeutet speziell, dass ,Effizienz um der Effizienz willen' kein Argument für die Erhöhung des Arbeitstempos sein kann. Eine als erforderlich erachtete Veränderung des Arbeitstempos muss ihre Rechtfertigung stattdessen durch eine über den Effizienzgedanken hinausgehende Darlegung erfahren. Je nach Sachlage kann es also angebracht sein, das Tempo zur Erledigung anstehender Aufgaben gut fundiert mal bewusst zu verringern oder mal bewusst zu erhöhen.

Wie aber kann der Akzelerationszirkel jeweils passend gesteuert werden? Der Vorschlag dieses Beitrags lautet, inn mittels geeigneter Moderatoren zu moderieren.

Zur Erläuterung soll auf das Bild des Stromgenerators zurückgegriffen werden. Man stelle sich den Akzelerationszirkel wie einen sogenannten moderierten Kernreaktor vor: indem die Brennstäbe des Reaktors mehr oder weniger tief in das als sogenannter Moderator eingesetzte schwere Wasser getaucht werden, wird die im Reaktor ablaufende Kettenreaktion immer auf das Level zwischen ,Stillstand" und ,ungebremst‘ (dem so genannten größten anzunehmenden Unfall - GAU) gebracht, auf dem dieser Stromgenerator gerade so viel Strom produziert, wie in einer gegebenen Situation erforderlich ist. Um dieses Bild auf den Akzelerationszirkel der sozialen Beschleunigung übertragen zu können, wird festgehalten, dass dieser „einschließlich seiner externen Motoren aus insgesamt neun Komponenten besteht, nämlich aus den drei externen Motoren, den drei Bereichen sozialer Beschleunigung und den drei Beziehungen (,Verbindungen') zwischen diesen Bereichen“ (Haack 2015: 271). Mithin sind analog zum Brennstab im Atomkraftwerk „neun ,Angriffspunkte' der Moderatoren im Beschleunigungssystem denkbar" (Abb. 2) 
(Haack 2015: 271), und es sind Instrumente analog dem schweren Wasser im Atomkraftwerk als Moderatoren zu suchen, mit deren Hilfe der Akzelerationszirkel über diese Angriffspunkte gesteuert - moderiert - werden kann.

\section{Agile Methoden als Moderatoren} des Akzelerationszirkels

\subsection{Ausgangspunkt ,Technische Re- daktion“}

Bei der anstehenden Suche nach Moderatoren des Akzelerationszirkels konzentrieren die Autoren sich zunächst ähnlich wie in wesentlichen Teilen der vorgehenden Überlegungen auf die Wirtschaft als Teilelement der Gesellschaft. Angesichts des vorliegenden Erfahrungshintergrundes und empirischen Materials (s. Abschnitt 5.2) wird konkret das durch Projekt- und Teamarbeit gekennzeichnete Arbeitsfeld Technischer Redakteurinnen und Redakteure fokussiert. Andere berufliche oder private Lebenszusammenhänge werden an dieser Stelle ausgeblendet. Anschließend kann wieder auf sie zurückgekommen werden, indem die gewonnenen $\mathrm{Er}$ gebnisse als Ausgangspunkt für einen Vorschlag zur Konstruktion von Moderatoren der sozialen Beschleunigung in allgemeinen Arbeits- und Lebenszusammenhängen genutzt werden (s. Abschnitt 5.3).

\subsection{Agile Prinzipien als Paradig- menwechsel in Technischen Redaktionen}

Zum Verständnis der Ansätze möglicher Moderatoren am Akzelerationszirkel der sozialen Beschleunigung bedarf es neuer Kriterien bei der Bemessung von Erfolg bei Projekt- und Teamarbeit, insbesondere in der Technischen Redaktion. Frei nach dem sokratischen Motto ,Du weißt, dass du nichts weißt!' wird gerade bei der Entwicklung von Produkten sowie bei der Erstellung ihrer Dokumentation das schnelle Lernen in den Fokus gerückt. Sogenannte Multiparametersysteme, wie sie heute in Produktentwicklung und Dokumentation vorgefunden werden können, reagieren sehr empfindlich auf das Zusammenwirken von Technologie,
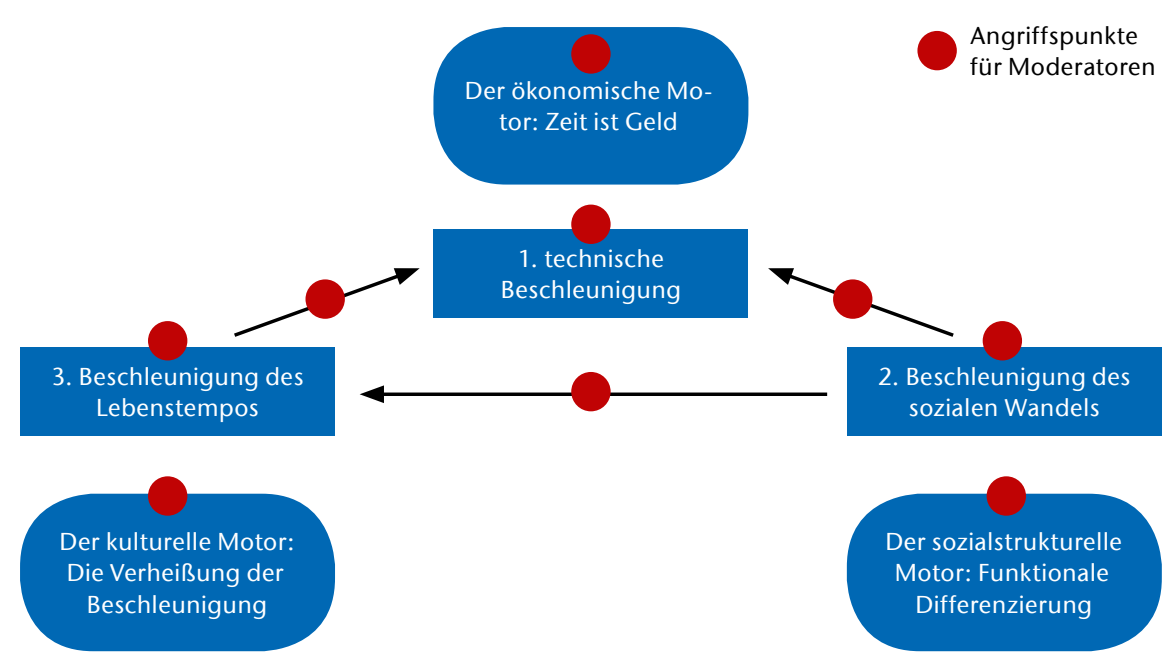

Abb. 2) Der moderierte Akzelerationszirkel der sozialen Beschleunigung (Haack \& Müller-Trabucchi 2016: 20, angelehnt an Rosa 2012: 309).

Märkten und Beteiligten. Ausgehend von der Komplexitätstheorie, wonach eine Vorhersage über den Erfolg in frühen Projektphasen unmöglich ist, liegt der Fokus also nicht mehr auf dem Vermeiden von Fehlern, sondern darauf, Entscheidungen über ,falsch oder ,richtig' vor dem Hintergrund der knappen Zeitressource zu einem möglichst frühen Zeitpunkt treffen zu können (vgl. Maurya 2012, Bartel \& Barbarski 2015). Essentiell dabei ist die von nahezu wissenschaftlicher Neugier und Sorgfalt getriebene Bereitschaft, Fehler zu machen. Scheitern gilt - so es denn rechtzeitig geschieht und damit zu einer Kurskorrektur führen kann - sogar als erwünschte Erfahrung. Das Ziel nachhaltigen Zeitmanagements ist es also nicht mehr, Prozesse ausschließlich zu beschleunigen, sondern teilweise sogar innezuhalten und, Wege rückwärts aus der Sackgasse‘ rechtzeitig anzutreten (vgl. Bartel \& Barbarski 2015).

Der notwendige Paradigmenwechsel in der Projekt- und Teamarbeit in Technischen Redaktionen besteht folglich darin, Probleme als lösbar zu betrachten, ohne den Lösungsweg und die Lösung bereits vorhersagen zu können. Gesucht sind also Rahmenbedingungen, die situationsgerechtes Handeln etwa im Sinne von Erkennen - Analysieren - Reagieren (antrainiertes Verhalten) oder im Sinne von Ausprobieren - Erkennen - Reagieren (lernendes Verhalten) erlauben (vgl. Haack \& Müller-Trabucchi 2016: 22).
Auf genau diesem Verständnis beruhen die Ansätze agiler Methoden: Basierend auf der Erfahrung, dass komplexe Projekte zu einem überdurchschnittlichen Prozentsatz scheitern, schufen Softwareentwickler 2001 eine Agenda, welche einen Wertekanon in das Zentrum des Handelns aller Beteiligten stellt, auf dessen Basis einzelne Prinzipien zur Bewältigung komplexer Aufgaben abgeleitet werden sollten: das Agile Manifest (Beck et al. 2001).

Abgeleitet aus den Prinzipien des Agilen Manifests entstanden vor der ganz undogmatischen Grundhaltung ,Agile is what works' in den vergangenen Jahren zahlreiche agile Methoden mit verschiedenen, über die Softwareentwicklung hinausgehenden, die Technischen Redaktionen bisher aber kaum berücksichtigenden Einsatzschwerpunkten. Allen Methoden gemein sind deren zugrundeliegende Werte hinsichtlich der Arbeit in interdisziplinären Teams sowie der Bereitschaft, auch auf unvorhergesehene Veränderungen kurzfristig reagieren zu können.

\subsection{Zeitmanagement-Workshops mit Technischen Redakteuren}

Vor diesem Hintergrund haben die Autoren dieses Beitrags im Frühjahr 2016 in insgesamt drei Workshops mit Technischen Redakteuren die Tauglichkeit agiler Methoden als Moderatoren für den o. g. Akzelerationszirkel diskutiert. Ziel war es, konkrete Maßnahmen auf der Basis agiler Me- 


\section{Individuen und Interaktionen}
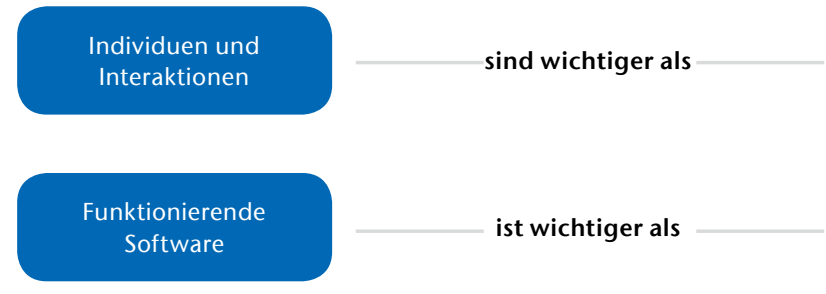

ist wichtiger als

Kooperation mit

Nutzern/Kunden

ist wichtiger als

Reaktion auf

Änderungen

ist wichtiger als

Abb. 3) Prinzipien des Agilen Manifests (Beck et al. 2001)

thoden zu erarbeiten, welche Potentiale zur Lösung der Zeitprobleme teaminterner und -übergreifender Zusammenarbeit in der Technischen Redaktion versprechen. Dazu wurden die Teilnehmerinnen und Teilnehmer der Workshops zunächst nach den aus ihrer Sicht wesentlichsten Ursachen für ihre Zeitprobleme befragt, um sie anschließend Lösungsansätze hierfür erarbeiten zu lassen.

Bemerkenswert ist, dass alle Workshops trotz der heterogenen Zusammensetzung der Teilnehmerinnen und Teilnehmer sowie trotz ihrer Unabhängigkeit voneinander nahezu identische Gründe für Zeitprobleme, aber auch überraschend übereinstimmende Bewertungen der Erfolgsquoten agiler Methoden als Moderatoren der sozialen Beschleunigung geliefert haben. Als bedeutendste Gründe für Zeitdruck und Zeitnot wurden das Multiprojektmanagement, mangelnde Priorisierung, unklare Aufgabendefinition, lückenhafte Informationsflüsse, spontane Zusatzaufgaben in späten Projektphasen und die wiederholt unzuverlässige Einschätzung von Arbeitsaufwänden genannt. Ebenso einhellig wurde die ,Selbstorganisation' von Teams, eine grundlegende Gemeinsamkeit agiler Methoden, als moderierend und damit als potentielle Lösung bei Zeitproblemen eingeschätzt. Transparenz, Kommunikation und regelmäßiger Wissenstransfer bilden als wesentliche Prinzipien der Organisation von Zusammenarbeit die entscheidende Basis für ein besseres, agiles Zeitmanagement in
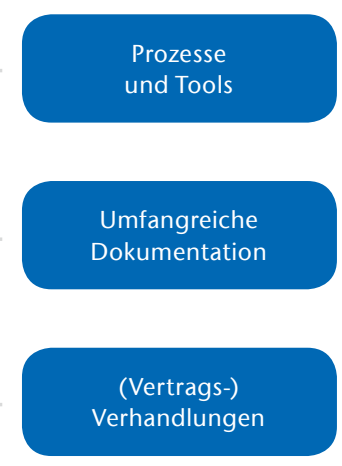

5.4 Moderation der sozialen Beschleunigung mit agilen Methoden

Die vorgenannten Methoden basieren durchgehend auf agilen Prinzipien. Sie belegen einerseits, dass die Idee der Moderation des Akzelerationszirkels in der täglichen Arbeit Technischer Redaktionen anwendbar ist, dass sie also nicht im Rang eines rein theoretischen und damit für praktische Belange weniger nützlichen Konstrukts verbleibt. Andererseits zeigen die Befunde, dass es agile Methoden gibt, die als Moderatoren der sozialen Beschleunigung und damit zum Zeitmanagement in den betrachteten Arbeitszusammenhängen in Technischen Redaktionen eingesetzt werden können.

Zusammenfassend liegen diesen agilen Methoden die gemeinsamen Werte ,Kommunikation', ,Ergebnisorientierung', ,Kollaboration' und ,Lernbereitschaft' zugrunde. Im Zusammenspiel mit den daraus abgeleiteten Prinzipien ,Anwenderorientierung", ,Interdisziplinarität', ,Simplicity', ,Visualisierung' und ,Perfektionismusverzicht' entsteht so ein Baukasten an Maßnahmen, welche durchgehend eine moderierende Wirkung auf Beschleunigungsprozesse ausüben. Dabei steht nicht die Effizienzsteigerung oder der Zeitgewinn des Einzelnen im Fokus, sondern stets der Erfolg von Teamprojekten - eine vor der Komplexität aktueller Projekte unverzichtbare Grundvoraussetzung.

Entsprechend lässt sich vermuten, dass die Moderation der sozialen Beschleunigung allgemein in beruflichen und privaten Kontexten - also nicht nur in Technischen Redaktionen - mit Hilfe agiler Methoden möglich ist.

In jedwedem Fall von Zeitverdichtung erscheint die Suche nach agilen Methoden als Moderatoren der sozialen Beschleunigung mithin als aussichtsreicher Versuch, der Zeitnot Herr zu werden. Hierfür schlagen die Autoren folgendes Vorgehen vor: dürfnissen der Anwender respektive ,Kunden‘ in Monetarisierungskontexten in den Fokus (vgl. Bartel \& Barbarski 2015), wurde in den Workshops aber ebenfalls als hilfreich aufgeführt.

1. Beschreibe und analysiere die vorliegende Situation unter Einbeziehung des Modells ,Akzelerationszirkel'.

2. Identifiziere die Angriffspunkte im Akzelerationszirkel, die sich für eine 
Moderation der sozialen Beschleunigung in der gegebenen Situation eignen.

3. Wähle eine oder mehrere bekannte Arbeitsmethoden aus oder konstruiere eine oder mehrere neue Methoden, die als Moderatoren an den ermittelten Angriffspunkten wirken könnten.

4. Falls es sich nicht bereits um agile Methoden handelt, modifiziere die Methoden aus 3. nach Möglichkeit so, dass sie den agilen Werten genügen.

5. Wende die agilen Methoden aus 4. an.

6. Wenn die Anwendung der Agilen Methoden aus 4. erfolgreich ist (also den Zeitdruck vermindert), speichere sie als Moderatoren für spätere Einsätze. Wenn die Nutzung der Methoden aus 4. nicht erfolgreich ist, laufe das Schema vollständig ( $a b$ 1.) oder in Teilen erneut durch bzw. brich es ab, sofern das Vorgehen aus guten Gründen aussichtslos erscheint.

\section{Fazit und Ausblick}

Ausgehend vom täglichen Erleben haben die Autoren ein grundsätzliches Zeitproblem konstatiert. Mehr und mehr fühlen Menschen sich unter Zeitdruck. Die Zeitnot entsteht durch Zeitverdichtung, die nicht mittels klassischer Zeitmanagementmethoden beseitigt werden kann, sondern durch diese eher noch verstärkt wird. Entsprechend ist es erforderlich, über das bekannte Zeitmanagement hinauszugehen und Vorschläge für den angemesseneren Umgang mit der eigenen Zeit zu entwickeln. Dies gelingt mit Blick auf Hartmut Rosas Theorie der sozialen Beschleunigung (Rosa 2012). Sie führt zu dem Vorschlag, nicht die Zeit zu managen, sondern die soziale Beschleunigung mit Hilfe von Moderatoren analog zum schweren Wasser in Atomkraftwerken zur Steuerung der Leistung dieser Kraftwerke zu beeinflussen und so zu moderieren.

Ergebnisse aus ZeitmanagementWorkshops mit Technischen Redakteurinnen und Redakteuren zeigen, dass das theoretische Konstrukt der Moderatoren brauchbar und anwendbar ist. Es wird daher vermutet, dass agile Methoden auch als Moderatoren der sozialen Beschleunigung in Lebens- und Arbeitszusammenhängen jenseits der Technischen Redaktionen geeignet sein können.

Konsequenz dieser Annahme ist ein aus sechs Schritten bestehendes Auswahl- und Konstruktionsschema für Moderatoren der sozialen Beschleunigung. Mit dessen Hilfe sollte es in der Zukunft gelingen, eine angemessene Zahl geeigneter Moderatoren für die soziale Beschleunigung zu entwickeln, so dass Zeitdruck und Zeitnot sowohl im Berufs- als auch im Arbeitsleben gemindert werden können. Insbesondere erlaubt das Schema, klassische Zeitmanagementmethoden beginnend mit Schritt 3 auf den Prüfstand zu stellen und, sofern möglich, im Sinne des Beyond-Zeitmanagement (Kapitel 2) zu Moderatoren für den Akzelerationszirkel auszuarbeiten (Schritte 4. bis 6.).

\section{LITERATUR}

Bartel D, Barbarski K (2015) Mit Customer Development durchstarten. Nach dem Original von Kevin Dewalt. Gründer.Guide Edition

Beck K, Beedle M, van Bennekum A, Cockburn A, Cunningham W, Fowler M, Grenning J, Highsmith J, Hunt A, Jeffries R, Kern J, Marick B, Martin RC, Mellor S, Schwaber K, Sutherland J, Thomas D (2001) Manifesto for Agile Software Development. http://agilemanifesto.org/. Accessed 19 Dec 2016

Covey SR (2014) Die 7 Wege zur Effektivität. Prinzipien für persönlichen und beruflichen Erfolg. GABAL Verlag, Offenbach. ISBN: 9783956230813

Haack B (2015) Beschleunigung moderieren statt Zeit managen. In: Biermann T (ed) Hochschulmanagement in Theorie und Praxis. Festschrift für László Ungvári. Wildau Verlag, Wildau, ISBN: 978-3-945560-03-7, pp 241-288

Haack B, Müller-Trabucchi M (2016) Wege aus der Zeitfalle. technische kommunikation 38(2):18-26

King V, Gerisch B (eds) (2009) Zeitgewinn und Selbstverlust. Folgen und Grenzen der Beschleunigung. CampusVerl., Frankfurt, M., New York, NY. ISBN: 9783593390291

Kodalle K-M, Rosa H (eds) (2008) Rasender Stillstand. Beschleunigung des Wirklichkeitswandels: Konsequenzen und Grenzen. Kritisches Jahrbuch der Philosophie, Bd. 12. Königshausen \& Neumann, Würzburg. ISBN: 9783826038266

Maurya A (2012) Running Lean. Iterate from Plan A to a Plan That Works, 1., neue Ausg. O'Reilly \& Associates, Sebastopol, CA. ISBN: 1449305172

Opitz F (2011) Speed. Auf der Suche nach der verlorenen Zeit, Orig.-Ausg., 1. Aufl. Riemann, München. ISBN: 9783570501283

Pichler R (2007) Scrum. Agiles Projektmanagement erfolgreich einsetzen. dpunkt, Heidelberg. ISBN: 978-389864-478-5
Rosa H (2012) Beschleunigung. Die Veränderung der Zeitstrukturen in der Moderne, 9. Aufl. Suhrkamp-Taschenbuch Wissenschaft, vol 1760. Suhrkamp, Frankfurt am Main. ISBN: 978-3-518-29360-7

Rosa H (2014) Beschleunigung und Entfremdung. Entwurf einer kritischen Theorie spätmoderner Zeitlichkeit, 3. Aufl. Suhrkamp, Berlin. ISBN: 3-518-58596-7

Rosa H (2015) Verdichtete Zeit. In: Le Monde diplomatique (ed) Atlas der Globalisierung. Weniger wird mehr. Der Postwachstumsatlas. Le Monde diplomatique. Taz-Verl.-und-Vertriebs-GmbH, Berlin, ISBN: 9783937683577, pp 42-43

Rosa H, Strecker D, Kottmann A (2013) Soziologische Theorien, 2., überarb. Aufl. utb-studi-e-book, vol 2836 UVK Verl.-Ges, Konstanz. ISBN: 978-3-8252-3832-2

Seiwert L (2005) Wenn du es eilig hast, gehe langsam. Das neue Zeitmanagement in einer beschleunigten Welt. Sieben Schritte zur Zeitsouveränität und Effektivität, 9., kompl. überarb. Aufl. Campus-Verl., Frankfurt/Main u. a. ISBN: 3-593-37665-2

Seiwert L (2009) Noch mehr Zeit für das Wesentliche. Zeitmanagement neu entdecken, 1. Aufl., überarb. u. aktual. Taschenbuchausg. Mosaik bei Goldmann. Goldmann Verlag, München. ISBN: 978-3-442-17059-3

Seiwert L (2013) Lass los und du bist Meister deiner Zeit. [mit Konfuzius entschleunigen und Lebensqualität gewinnen], 1. Aufl. GU Lebensführung. Gräfe u. Unzer, München. ISBN: 9783833833908

Wolf $\mathrm{H}$, Andrezak M (eds) (2012) Agile Projekte mit Scrum, XP und Kanban im Unternehmen durchführen. Erfahrungsberichte aus der Praxis, 1. Aufl. dpunkt.verl., Heidelberg. ISBN: 9783898647526

\section{AUTOREN}

Prof. Dr. Bertil Haack

Dekan Fachbereich Wirtschaft, Informatik, Recht Technische Hochschule Wildau

Markus Müller-Trabucchi

Niederlassungsleiter

DMK E-Business

E-Mail für Korrespondenz: bertil.haack@th-wildau.de

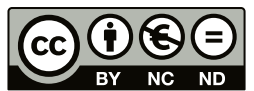

